\title{
Competência interpessoal no cuidado de pessoas com diabetes: percepção de enfermeiros
}

\author{
Interpersonal competence in caring of people with diabetes: perception of nurses
}

Competencia interpersonal en el cuidado de personas con diabetes: percepción de los enfermeros

\author{
Daniela Arruda Soares', Dora Sadigursky", Isabela Soares"I' \\ ' Universidade Federal da Bahia, Instituto Multidisciplinar em Saúde, \\ Núcleo de Epidemiologia e Saúde Coletiva. Salvador-BA, Brasil. \\ "Universidade Federal da Bahia, Escola de Enfermagem, \\ Disciplina Enfermagem Psiquiátrica e Saúde Mental. Salvador-BA, Brasil. \\ III Universidade Federal da Bahia, Instituto de Saúde Coletiva. (Mestranda) Salvador-BA, Brasil.
}

Submissão: 8/2/2010 Revisão: 7/11/2010 Aprovação: 12/12/2010

\section{RESUMO}

Pesquisa de natureza qualitativa e exploratória que objetivou apreender a percepção de enfermeiros que cuidam de pessoas com Diabetes sobre a competência interpessoal. Os sujeitos foram onze enfermeiros que exerciam suas atividades em Unidades de Saúde da Família da zona urbana e que preencheram um termo de consentimento. Os dados, obtidos por entrevista semiestruturada, foram analisados e categorizados por meio de análise temática. Os resultados apontaram que os enfermeiros percebem a competência interpessoal como uma habilidade de interação com o paciente, como estabelecimento de um relacionamento interpessoal efetivo e como forma de relacionamento interpessoal. Depreende-se que o exercício desta competência não é passível de padronização, o que enfatiza a singularidade dos processos de interação e do cuidado à saúde. Descritores: Enfermagem; Competência profissional; Relações interpessoais.

\section{ABSTRACT}

This is a qualitative and exploratory study which aimed to apprehend the perceptions of nurses who care for people with Diabetes on the interpersonal competence. The subjects were eleven nurses who performed their activities in the Units of Family Health in the urban area, and completed a consent form. The data, obtained through semi-structured interview, were analyzed and categorized by thematic analysis. The results showed that nurses perceive the interpersonal competence as an ability to interact with the patient, as the establishment of an effective interpersonal relationship and as forms of interpersonal relationships. It appeared that the exercise of that power is not subject to standardization, what emphasizes the uniqueness of the processes of interaction and of health care.

Key words: Nursing; Professional competence; Interpersonal relationship.

\section{RESUMEN}

Estudio de investigación cualitativo y exploratorio que objetivó aprehender las percepciones de enfermeros que cuidan a personas con Diabetes sobre competencia interpersonal. Los sujetos fueron once enfermeros que realizaban sus actividades en Unidades de Salud de la Familia en el área urbana y completaran el formulario de consentimiento. Los datos, recogidos a través de entrevistas semi-estructuradas, fueron analizados y categorizados por el análisis temático. Los resultados mostraron que las enfermeras perciben la competencia interpersonal como la capacidad de interactuar con el paciente, tales como el establecimiento de una relación interpersonal eficaz y como formas de relaciones interpersonales. Parece que el ejercicio de esta facultad no está sujeto a la normalización, que hace hincapié en la singularidad de los procesos de interacción y atención de la salud.

Palabras clave: Enfermería; Competencia profesional; Relaciones interpersonales. 


\section{INTRODUÇÃO}

A discussão e a busca pelo desenvolvimento de competências profissionais para atuação no âmbito da saúde encontra ressonância nas modificações funcionais e estruturais sofridas por este setor, refletindo uma dinamicidade e complexidade dos processos, bem como a necessidade dos profissionais de saúde de desenvolverem uma práxis comprometida com esta realidade.

No tocante à profissão de enfermagem, o cuidado direto prestado aos indivíduos no ciclo saúde versus doença, o engajamento sanitário, político e social desta profissão, seja nos contextos local, estadual ou federal para a construção e efetivação do Sistema Único de Saúde (SUS), tem direcionado à realização de estudos implicados com o referencial das competências em distintos contextos ${ }^{(1-5)}$.

Pensar em competências gerenciais ${ }^{(6)}$, competências essenciais para atuação na saúde pública ${ }^{(5)}$, competências para o cuidar $^{(7)}$, competências sociais e políticas ${ }^{(8)}$, ou competências para o relacionar ${ }^{(2,9)}$, implica em reconhecer as competências como uma forma de tecnologia para o trabalho em saúde, uma ferramenta que subsidia ações em saúde mais participativas e democráticas, além de instrumentalizar os enfermeiros a transitarem da alienação, do imobilismo e do tecnicismo, para o exercício criativo, reflexivo, crítico e competente da sua profissão no contexto da assistência à saúde.

Entre os tipos de competências que vêm sendo delineadas e desenvolvidas pelos enfermeiros, destaca-se a competência interpessoal. Este destaque não objetiva considerá-la como a única competência capaz de dar conta de todas as ordens de problemas evidenciados pelo setor saúde, muito menos pelos que são enfrentados por enfermeiros durante o seu exercício profissional, mas, sim, porque a mesma pode concorrer para a humanização das relações de trabalho e do próprio cuidado, além de viabilizar o desenvolvimento de novas competências e o enfrentamento de desafios.

Tomando como base o entendimento da competência interpessoal como a "habilidade de lidar eficazmente com as relações interpessoais" ${ }^{\prime \prime(10)}$, espera-se que a mesma viabilize a implementação de práticas de cuidado que relativizem o poder, as estratégias de dominação, abrindo espaço para a emergência de princípios como o diálogo, a co-responsabilidade, o pluralismo, a alteridade, o questionamento das práticas, a saída da retórica e a implicação das práticas de cuidado compatíveis como o contexto em que estas se desenrolam ${ }^{(11)}$.

Isto posto para o cuidado de pessoas que vivenciam situações de enfermidades orgânicas tal como o Diabetes, doença que exige do enfermo mudanças abrangentes em sua maneira de viver a vida, a competência interpessoal poderá contribuir de maneira decisiva, incrementando as práticas de cuidado, no sentido de evitar que o paciente tenha complicações a curto e longo prazo e, em grau extremo, o comprometimento da vida.

Diante do exposto, questiona-se: qual a percepção de enfermeiros que cuidam de pessoas com Diabetes sobre competência interpessoal? Na tentativa de buscar respostas para este questionamento, o presente trabalho tem como objetivo apreender a percepção de enfermeiros que cuidam de pessoas portadoras de Diabetes sobre a Competência Interpessoal.

\section{A COMPETÊNCIA INTERPESSOAL E O CUIDADO DE PESSOAS COM DIABETES}

"A ação de cuidar exige um olhar atento e está inserido numa dinâmica dialética entre o cuidador e aquele que é cuidado"(12). Esta dinâmica imprime às relações interpessoais um sentido ontológico e epistemológico e as apontam como inerentes à existência humana, portanto ao cuidado em saúde, tornando-as capazes de interferirem nos estados de saúde e doença.

Neste sentido, vislumbra-se a habilidade para as relações interpessoais como uma ferramenta estratégica capaz de viabilizar não somente a aplicação das tecnologias disponíveis para alcançar a saúde, mas o exame da relação entre finalidades e meios e seu sentido prático, conforme um diálogo o mais simétrico possível entre profissionais e usuários dos serviços ${ }^{(13)}$.

Pensar nas relações interpessoais como ferramenta capaz de auxiliar na superação das tradicionais formas de cuidar pautadas no êxito técnico, no conhecimento aprisionado no método ${ }^{(14)}$, no distanciamento entre profissionais de saúde e usuários dos serviços, neste caso, entre enfermeiros e pessoas com Diabetes, remonta a necessidade de compreensão das características das relações interpessoais, suas implicações no cuidado de pessoas acometidas pelo Diabetes e sua relação com a competência interpessoal.

Sob este prisma destaca-se que as relações interpessoais são imateriais, pois, são produzidas e concomitantemente consumidas pelos envolvidos ${ }^{(15)}$. Elas são designadas na enfermagem $^{(16)}$ de relação pessoa-a-pessoa, representando a relação entre a enfermeira e o ser cuidado, evidenciando um processo em que ambos podem compartilhar seus sentimentos, valores e significados e perceberem a singularidade um do outro, transcendendo os papéis desempenhados. Também é denominada de relação de ajuda ou terapêutica ${ }^{(17)}$, acrescentando que por meio dela pode-se concorrer para que a pessoa atendida aceite a si mesmo e a própria doença, viabilizando a mudança de comportamento e o estabelecimento de uma comunicação efetiva.

Salienta-se que a relação pessoa-a-pessoa / terapêutica é inerente ao cuidado em saúde, bem como que o cuidar é parte integrante da vida, pois, ninguém vive sem cuidado ${ }^{(18)}$. Portanto, as pessoas com Diabetes quando em interação com o profissional enfermeiro, necessariamente, requerem que o relacionamento estabelecido com as mesmas seja terapêutico, expressando uma forma de cuidar subjacente que o atenda em suas necessidades de saúde, pois, muitas vezes, apresentam atitudes de negação, recusa, revolta, raiva, barganha, tristeza, depressão ou aceitação diante da doença ${ }^{(19)}$.

Desta forma, quando se fala da necessidade da competência interpessoal perpassar pelo cuidado de pessoas com Diabetes, refere-se não apenas a tratar essas pessoas no sentido de estarem com uma doença crônica e de melhorar a sintomatologia proveniente da mesma, mas sim, a lançar um novo olhar sobre estas, buscando compreender o significado da doença, os itinerários terapêuticos percorridos frente à manutenção da saúde ou em situação de doença, considerando o contexto em que 
estas pessoas estão inseridas, suas crenças, aspirações, cultura, estimulando a sua participação ativa neste processo, por meio de uma relação intersubjetiva de saberes e fazeres.

Por isso, o desenvolvimento de competência interpessoal com vistas à manutenção de um relacionamento interpessoal harmonioso, viabilizará maior sinergismo entre as relações de trabalho e, sobretudo com as pessoas portadoras de Diabetes, na tentativa de equilibrar a mobilização de competência técnica e de competência interpessoal.

Além disso, a utilização da competência interpessoal nestas circunstâncias pode subsidiar a identificação de intervenções mais efetivas capazes de melhorar a adesão do paciente às orientações e ao tratamento e, por conseguinte, melhorar o controle glicêmico e prevenir complicações agudas e crônicas.

Contudo, para que se possa desenvolver a competência interpessoal e utilizá-la como uma tecnologia no cuidado à saúde, faz-se mister compreender os sentidos teóricos que lhe são atribuídos com vista ao apontamento de indícios que reflitam a práxis cuidativa / relacional bem como os resultados oriundos da mesma.

Em consonância com o exposto, entende-se que o termo competência constitui-se em uma noção polissêmica que envolve várias acepções e abordagens ${ }^{(20)}$. Começou a ser utilizado na Europa, na década de 1980, constituindo a base das políticas para formação e capacitação de trabalhadores.

As definições de competências enfocam, em essência, a condição de aplicação adequada de conhecimentos, habilidades e destrezas, juntamente com atitudes e compromissos, bem como a capacidade integral de exercer uma atividade, possibilitando aos indivíduos uma participação consciente e crítica no âmbito do trabalho, bem como sua autorrealização(5,20).

Da concepção ampliada de competência para a delimitação do conceito de competência interpessoal, encontra-se esta como o resultado de "habilidades específicas comportamentais que conduzem a conseqüências significativas no relacionamento duradouro e autêntico, satisfatório para as pessoas envolvidas"(10).

Tanto na macro definição sobre competência, quanto na definição de um tipo específico de competência como a interpessoal, percebe-se a necessidade de reconhecer as diferentes visões que os profissionais enfermeiros têm sobre competência interpessoal, pois, neste trajeto, coloca-se em pauta a redefinição do papel do profissional (não somente como aplicador de técnicas), a revalorização da posição da pessoa diabética no contexto do cuidado (não apenas passiva a prática assistencial) e a inclusão da competência interpessoal ( não só para relativizar o poder do saber e do fazer, mas também para diminuir a apartação dos usuários em relação aos serviços de saúde bem como da sua própria saúde).

\section{METODOLOGIA}

Trata-se de um estudo de caráter descritivo e exploratório com abordagem qualitativa. O locus do estudo foi constituído por onze Unidades de Saúde da Família (USF) situadas em um município do sudoeste baiano, o qual é considerado o principal pólo regional de prestação de serviços nas áreas de educação e saúde da região. As unidades foram escolhidas aleatoriamente, porém priorizaram-se as da zona urbana, devido à facilidade de acesso e ao maior número de profissionais nas mesmas.

A amostra foi constituída de onze enfermeiros que preencheram os seguintes critérios de inclusão: trabalhavam nas unidades de saúde família da zona urbana do município, desenvolviam o atendimento às pessoas com Diabetes, preencheram o termo de consentimento livre e esclarecido.

Considerando a natureza deste estudo, o conteúdo das falas e a participação dos sujeitos foi encerrada a partir do momento em que se percebeu que as respostas emitidas apresentaram uma saturação teórica dando clareza para a compreensão do objeto da pesquisa, assim como refletindo a totalidade nas suas múltiplas dimensões ${ }^{(21)}$.

Foram seguidos todos os procedimentos éticos exarados pela Resolução 196/96 do Conselho Nacional de Saúde / Ministério da Saúde, sendo o projeto do estudo aprovado pelo Comitê de Ética em Pesquisa da Universidade Estadual do Sudoeste da Bahia, pelo Processo n. ${ }^{\circ}$ 38/2006.

Para a obtenção dos dados foi utilizada a entrevista semiestruturada, com uma questão norteadora que versava sobre a percepção de enfermeiros que cuidam de pessoas com Diabetes sobre competência interpessoal.

O período de coleta dos dados compreendeu os meses de março a junho de 2006. Salienta-se que as entrevistas foram realizadas nas Unidades de Saúde da Família, na sala de atendimento do próprio profissional, e geralmente ocorriam no final do expediente de trabalho.

O conteúdo das entrevistas foi categorizado seguindo a técnica de análise de conteúdo, modalidade temática ${ }^{(22)}$ a qual compreende três etapas, a saber: pré-análise, exploração do material e tratamento dos dados obtidos e interpretação.

$\mathrm{Na}$ pré-análise foi realizada a leitura flutuante do material pesquisado, no sentido de constituir um corpus, o qual representou a organização do material coletado. Na fase de exploração do material buscou-se codificar o núcleo de compreensão do mesmo, agregando dados e informações, bem como selecionando as categorias teóricas ou empíricas que nortearam a especificação do tema. Essa categorização foi realizada por meio do desmembramento do texto em unidades temáticas, seguida de reagrupamentos analógicos. Por fim, na última fase, propôs-se inferências e realizou-se interpretações fundamentadas no quadro teórico delineado neste trabalho, as quais estão descritivamente tratadas na etapa que se segue.

\section{APRESENTAÇÃO E DISCUSSÃO DOS RESULTADOS}

As falas dos enfermeiros deste estudo acerca da percepção da competência interpessoal no cuidado de pessoas com Diabetes fizeram emergir duas categorias, descritas a seguir.

\section{Categoria1- Habilidades/capacidade de interação com o paciente}

Os enfermeiros apontaram que a percepção que têm sobre competência interpessoal significa possuir habilidade/capacidade para interagir com o paciente. Tal fato pode ser ratificado nas falas seguintes: 
Habilidade de estabelecer interação apesar das diferenças existentes quanto a ideias, valores e crenças. (ENF 5)

Saber ou ter habilidade... de entender os outros respeitando as particularidades de cada um e buscar harmonia entre os atores sociais envolvidos no meio que se apresenta. ( ENF 1)

... Habilidade de interagir com o outro, neste caso o portador de Diabetes, ajudando-o a melhorar sua qualidade de vida através da prestação de uma assistência voltada para a promoção da saúde e prevenção de complicações. ( ENF 11)

A habilidade e conhecimentos específicos para podermos relacionar com o outro, de modo que a gente possa cuidar dos nossos pacientes. (ENF 6)

É possível perceber uma correlação entre os conteúdos das falas e o que é abordado em alguns estudos na área ${ }^{(2,8,10,20,23-26)}$. Todos fazem apologia à competência interpessoal como sinônimo de detenção de habilidades, dentre elas as relacionais e como capacidade para realização de uma determinada atividade, assim como para se relacionar com o outro.

Os conceitos genéricos sobre competência ainda se apóiam no tripé "saber, saber-fazer e saber-ser, ou a conhecimento, habilidade e atitude"(27), os quais devem estar associados à capacidade de solução de problemas.

Esta compreensão sobre competência interpessoal foi recorrente em grande parte das falas dos enfermeiros, evidenciando que a competência interpessoal jamais deve permitir a negação do outro, dos seus problemas, crenças, desejos ou aspirações em detrimento da imposição de conhecimentos e desejos do cuidador. Ao contrário, a busca deve ser pela harmonização dos interesses e expectativas de ambos os envolvidos no processo cuidativo/relacional.

A competência interpessoal visa à valorização da subjetividade do enfermeiro e da pessoa portadora de Diabetes, "portanto, não considera apenas a utilização racional de conhecimentos, mas a sua mobilização de acordo com a situação e a subjetividade de cada um"(2).

Pode-se depreender que não é suficiente manejar uma morbidade mediante o seguimento de normas e rotinas protocolares, é necessário saber interagir para cuidar do indivíduo consoante as suas demandas, é preciso ainda encarar o portador do Diabetes como um sujeito social, "como um ser que produz sua própria história, o responsável pelo seu devir,... sujeito de sua própria saúde"(13).

Não obstante, reconhece-se que habilidades e atitudes específicas implícitas às relações interpessoais, refletem também a capacidade para cuidar do outro, sendo este cuidado de enfermagem configurado como um processo interativo que conecta o ser que cuida ao ser cuidado.

Assim, o cuidado é percebido como uma atividade dinâmica, reflexiva, pautada na interação humana ${ }^{(28)}$. Acrescenta-se, ainda, que o mesmo se constitui em uma relação representada por sentimentos e por um interesse genuíno para promover o bem-estar do outro.
Desta forma, é interessante perceber que a competência interpessoal foi apontada, por alguns informantes, como forma de subsidiar o cuidado de enfermagem. Eles mencionaram que a habilidade de se relacionar bem possibilita cuidar meIhor das pessoas com Diabetes.

Há que se considerar a competência interpessoal como um elemento propulsor e redirecionador das práticas de saúde e do cuidado de enfermagem, como um "pano de fundo, onde se desdobram as demais competências"(2), como a mobilização de habilidades, hábitos e atitudes para soluções de situações concretas e/ou imprevisíveis de trabalho, como a possibilidade de interpretação de signos e significados das enfermidades no sentido de contribuir para um cuidado mais humano, ético, efetivo e emancipador ${ }^{(8)}$.

A competência interpessoal, portanto, denota habilidades de interação com o outro, mas não se limita a isto. Seu conceito mais amplo implica a consideração de comportamentos, de atitudes, de percepção, de conhecimento científico, de inteligência, de valores, de emoções e sentimentos postos em prática, nas situações concretas de trabalho e cuidado.

\section{Categoria 2- Relacionamento interpessoal terapêutico}

Além de perceberem a competência interpessoal como sinônimo de habilidades de interação com o paciente, os enfermeiros deste estudo ainda apontaram o relacionamento interpessoal terapêutico como a expressão desta competência.

O relacionamento interpessoal terapêutico é caracterizado como um fluxo experiencial no qual o terapeuta e o cliente participam por meio de uma relação intersubjetiva, com vistas ao benefício do cliente ${ }^{(15)}$. As falas abaixo refletem o exposto:

Capacidade que a pessoa tem de conseguir uma relação interpessoal efetiva. (ENF 4)

Desenvolvimento de relações efetivas entre as pessoas de forma cordial, espontânea, atenciosa, sensata e sobretudo transmitindo-Ihe amor e segurança, a fim de que se estabeleça uma relação de confiança entre ambas as partes. (ENF 2)

É a interação efetiva entre dois sujeitos, ou seja, é a troca de informações entre duas pessoas de modo que haja o entendimento entre ambas. (ENF 10)

É quando nós conseguimos nos relacionar bem com os pacientes superando as nossas expectativas e as do próprio paciente. (ENF 9)

As relações interpessoais, de fato, compõem a competência interpessoal, e são de certo modo reflexos da mesma. Contudo, reconhecer que uma pessoa é competente porque desenvolveu um relacionamento interpessoal eficaz, vai além de dizer que ela estudou e pode dar conta, teoricamente, do que aprendeu. Isto porque "a qualificação real do trabalhador, compreendida como um conjunto de competências..., vão além da dimensão cognitiva"(29) refletindo também a dimensão da inteligência prática e a compreensiva. 
Nesta ótica, se relacionar de forma efetiva com o outro reflete muito mais o saber-ser e saber-agir do que o saber-fazer. Representa que a subjetividade, os saberes tácitos, os comportamentos, as atitudes frente a situações concretas de relacionamento interpessoal, tem forte peso e influência.

Entretanto, uma preocupação deve ser expressa, no sentido de não se recair na falácia de demonizar as técnicas, centradas no saber-fazer, e nem de enfatizar o empirismo, ressaltando o saber-ser e o agir, mas sim de destacar que não existem fórmulas prontas para se alcançar um relacionamento interpessoal efetivo, visto que o próprio conceito de competência vincula-se à mobilização de habilidades e conhecimento para lidar com o imprevisível, reduzindo a excessiva instrumentalidade e o caráter prescritivo, característicos de outros modelos tradicionais de cuidar e relacionar.

Ainda no que diz respeito aos relacionamentos interpessoais, foi mencionado em uma das falas, que este ocorre quando as expectativas do enfermeiro e da pessoa enferma são superadas. De fato, quando ocorre a superação de expectativas de ambos, a sensação de gratificação para o ego é maior. Embora seja gratificante, nem sempre o enfermeiro superará suas expectativas, especialmente em um primeiro momento de contato com o paciente. Isto porque o profissional, como ser humano, pode ter enfrentado problemas em sua trajetória de vida, resultando em obstáculos ao estabelecer uma relação com os pacientes ${ }^{(16)}$.

Assim, conhecer-se para conhecer o outro e poder ajudá-lo, implica em um movimento de fortalecimento do EU do profissional de enfermagem e da pessoa enferma. Sob este prisma é que "identificamos as variáveis individuais e organizacionais na construção da competência interpessoal, como instrumento do trabalho em saúde"(2). Em outros termos, as variáveis individuais, representativas da subjetividade do enfermeiro e do paciente, não podem ser deixadas à margem quando se fala de competência interpessoal, pois, fazem parte do trabalho em saúde e do cuidado de enfermagem.

\section{Categoria 3- Formas de relacionamento interpessoal}

As falas agrupadas nesta categoria revelam que existem formas distintas de relacionamento interpessoal, e que tais formas, quando utilizadas pelo enfermeiro, denotam a competência interpessoal.

Estas formas de relacionamento interpessoal, apontadas pelos enfermeiros como uma espécie de certificação da competência interpessoal, diferentemente da certificação ocupacional, promovida ou requerida por empresas, não possuem, necessariamente, uma equivalência com títulos educacionais que respondem exclusivamente às leis do mercado ${ }^{(30)}$.

Isto porque aspectos como confiança, respeito, interesse, compreensão, conforto, apesar de serem implícitos às questões éticas que permeiam o cuidado em saúde, são vistos, geralmente, como secundários, quando não acessórios deste cuidado, tanto no âmbito da formação quanto da assistência à saúde, refletindo uma marca indelével centrada na técnica e na produtividade.

No entanto, a menção destes aspectos por parte dos enfermeiros participantes deste estudo, já demonstra certa ruptura quanto a estes paradigmas biotécnicos, bem como acerca do conceito de competência interpessoal e da sua operacionalização, tal como se segue abaixo.

Me relaciono de forma prestativa, dispensando-lhe atenção e transmitindo confiança. (ENF 2)

Ter competência interpessoal é procurar me relacionar por meio do desenvolvimento da confiança, respeito e conhecimento técnico e científico. (ENF 4)

Buscando oferecer conforto, confiança e resolver, na medida do possível, seus problemas de saúde. (ENF 9)

Além do aspecto profissional de cuidado técnico, procuro estabelecer um vínculo de confiança em que o cliente possa expor seus medos, tire suas dúvidas. (ENF 3)

A competência interpessoal representa o relacionamento cordial baseado no respeito quanto às crenças, valores adquiridos, mas sem perder de vista as questões científicas referentes ao problema citado. ( ENF 5).

Considerando que o conceito de competência engloba a capacidade de mobilização do saber-fazer, saber-ser e saber-agir, tais falas comportam também, uma visão ainda fragmentada da competência interpessoal, colocando na arena do debate a indissociabilidade entre os modos de fazer/ relacionar/ intervir no contexto do trabalho em saúde onde as ações de cuidar são desenvolvidas.

Nesta direção, observa-se nas falas certa cisão entre a técnica - representando o pólo objetivo, supostamente ancorada exclusivamente em conhecimentos científicos -, e a ética refletindo o pólo subjetivo, compreendida como da ordem relacional, dos vínculos entre profissionais e usuários ${ }^{(31)}-$, implicado em uma necessidade de união entre estes pólos, tendo na competência interpessoal este elemento integrativo, para que o cuidado não se torne apenas um instrumental de caráter repetitivo e dominador.

Sob este ponto de vista, o enfermeiro 3 afirmou que, além do aspecto profissional do cuidado técnico, procura estabelecer confiança, abertura do paciente para exposição dos medos e de dúvidas. Tal conteúdo também ficou expresso nas falas dos enfermeiros 4 e 5 .

Nesta direção, estes enfermeiros enfatizaram o conhecimento científico sem, no entanto, perderem de vista que as distintas formas de se relacionarem com pessoas com Diabetes são também integrantes dos eixos que norteiam o cuidado em saúde a estas pessoas.

Em consonância com o exposto, destaca-se que "o cuidar apresenta uma conotação diferente ao não se limitar apenas ao aspecto técnico e a realização de uma tarefa ou procedimento" ${ }^{\prime(32)}$. Além disso, o cuidar possui um componente moral e emocional, o aspecto cognitivo, a percepção, o conhecimento e a intuição.

Os aspectos técnicos devem ser valorizados, mas eles por si só não garantem a adesão dos pacientes ao regime 
terapêutico, não garantem que as informações fornecidas pelo enfermeiro nas consultas, visitas domiciliares ou grupos de educação em saúde sejam incorporados pelo paciente gerando hábitos de vida saudáveis. Isto porque, a valorização da sensibilidade e da interação afetiva "propicia a compreensão e modificação das pessoas mais do que um raciocínio brilhante repassado mecanicamente" ${ }^{\prime \prime(34)}$.

Complementarmente, também foi apontado pela maioria dos entrevistados a confiança e o respeito como elementos imprescindíveis para o desenvolvimento do relacionamento. A confiança pode ser definida como a "qualidade que se desenvolve através de relações de respeito, segurança e honestidade $^{\prime \prime(32)}$. Chama-se atenção para que esta qualidade não culmine em relações paternalistas, que provoquem dependência.

No entanto, a capacitação do indivíduo, com vistas à construção de atitudes positivas e a transformação do comportamento, faz cair por terra o paternalismo imobilizador em relação ao indivíduo com Diabetes, pois, ao contrário, a relação interpessoal busca a responsabilização e autonomização do mesmo pela sua saúde, pela superação do estado de doença e pelas suas situações de vida.

Faz-se necessário esclarecer ainda que existem formas de relacionamento interpessoal, e não fórmulas prontas, capazes de conduzir as pessoas a situações desejadas.

\section{CONSIDERAÇÕES FINAIS}

As percepções de enfermeiros acerca da competência interpessoal fundamentaram-se em habilidades de interação com o paciente, no estabelecimento de relacionamento interpessoal efetivo e em formas de relacionamento interpessoal.
Tais percepções se coadunaram grandemente com as proposições teóricas acerca da competência interpessoal, entremeadas com a ênfase do conhecimento técnico-científico como fator preponderante para a execução, de maneira facilitada, dos relacionamentos interpessoais.

Ressalta-se que este conhecimento é importante e deve ser considerado, mas, em suas devidas proporções para que não descambe em mero tecnicismo e reificação dos sujeitos com Diabetes.

Destaca-se ainda que, a contribuição do estudo por meio destas reflexões sobre competência interpessoal, reside no fato de preencher algumas lacunas a respeito de um conceito relativamente novo e complexo e que necessita de maior discussão e debate no âmbito da saúde.

Para que ocorra o trânsito das percepções de enfermeiros sobre competência interpessoal para a prática, faz-se mister mudanças nos paradigmas em que estão ancorados o cuidar e o relacionar, o que a princípio pode gerar certa insegurança e resistência por parte destes profissionais, já que o caminho indicativo para o exercício desta competência, não está predito em protocolos de abordagem às doenças, comumente encontrados nas Unidades, o que enfatiza a singularidade dos processos de interação e do cuidado à saúde. No entanto, destaca-se que a mesma pode ser aprendida e desenvolvida pelos enfermeiros.

O referencial das competências pode potencializar as ações de enfermagem frente às pessoas com Diabetes e a todas as ações desenvolvidas pelo enfermeiro, contribuindo, desta forma, para o congraçamento de propostas que visem o crescimento desta profissão e a melhoria da qualidade de vida dos sujeitos foco desses cuidados.

\section{REFERÊNCIAS}

1. Nery AA. A prática do enfermeiro instrutor/supervisor do Programa de Agentes Comunitários de Saúde: uma questão de competência interpessoal [Dissertação]. Rio de Janeiro (RJ): Escola Enfermagem de Rio de Janeiro, Universidade do Rio de Janeiro; 1998. Mestrado em Enfermagem.

2. Barduchi RI. Compreendendo a competência interpessoal na vivência do ser-docente no contexto relacional com o aluno de graduação [tese]. Ribeirão Preto (SP): Escola de enfermagem de Ribeirão Preto, Universidade de São Paulo; 2002. Doutorado em Enfermagem.

3. Fernades JD, Araújo FA, Fernandes J, Reis LS, Gusmão MCC, Santana N. Competência interpesssoal nas práticas de saúde: o individual e o coletivo organizacional. Texto Contexto Enferm 2003;12:(2) 201-215.

4. Murani DB, Bezerra AL. Inclusão da competência interpessoal na formação do enfermeiro como gestor. Rev Bras Enferm 2004;57(4):350-356.

5. Wiit RR. Competência da enfermeira na atenção básica: contribuições à construção das Funções Essenciais de Saúde Pública [tese]. Ribeirão Preto (SP): Escola de
Enfermagem de Ribeirão Preto, Universidade de São Paulo; 2005. Doutorado em Enfermagem.

6. Peres AM, Ciampone MHT. Gerência e competências gerais do enfermeiro. Texto Contexto Enferm 2006;15 (3):492-9.

7. Sena RR, Silva KL, Gonçalves AM, Duarte ED, Coelho S. O cuidado no trabalho em saúde: implicações para a formação dos enfermeiros. Interface Comunic Saúde Educ 2008;12(24):23-4.

8. Zarifian PA. Gestão da e pela competência. In: Anais do Seminário Internacional de Educação profissional, trabaIho e competência; 1998 jan; Rio de Janeiro (RJ), Brasil. Rio de Janeiro: SENAI/DN-CIET; p.120-230.

9. Soares DA, Sadigursky D. A competência interpessoal como elemento para o cuidado de enfermagem. Rev Saúde.com 2009;5(2):30-20.

10. Moscovici F. Desenvolvimento Interpessoal. Rio de Janeiro: José Olympio; 1996.

11. Foucalt M. A hermenêutica do sujeito. São Paulo: Martins Fontes; 2006. 
12. Fukumitsu KO, Cavalcante F, Borges MS. O cuidado na Saúde e na doença: uma perspectiva gestáltica. Rev Estu Pesq Psicol 2009;9(1):172-82.

13. Ayres JRCM. Cuidado e reconstrução das práticas de saúde. Interface - Comunic Saúde Educ 2004;8(14):73-91.

14. Pinheiro R, Guizardi F. Cuidado e Integralidade: Genealogia de Saberes e Práticas no Quotidiano. In: Pinheiro R, Mattos RA, organizadores. Cuidado as fronteiras da integralidade. Rio de Janeiro: CEPESC/UERJ, ABRASCO; 2006. p. 21-36.

15. Wood JK. Abordagem centrada na pessoa. 3a ed. Vitória: Fundação Ceciliano Abel de Almeida; 1997.

16. Travelbee J. Intervención em enfermería psiquiátrica: el processo de lá relación de persona a persona. Cali: Carvajal; 1979.

17. Furegato ARF. Relações interpessoais na Enfermagem. Ribeirão Preto- SP: Scala; 1999.

18. Coliére MF. Promover a vida. Lisboa: Lidel; 1999.

19. Sandoval RCB. Grupo de convivência de pessoas com Diabetes mellitus e familiares: percepção acerca das complicações crônicas e conseqüências sociais crônicas [dissertação]. Florianópolis (SC): Escola de Enfermagem de Florianópolis, Universidade Federal de Santa Catarina; 2003. Mestrado em Enfermagem.

20. Deluiz N. Qualificação, competências e certificação: visão do mundo do trabalho. In: Ministério da Saúde (BR). Humanizar cuidados de saúde: uma questão de competência. Brasília: Ministério da Saúde; 2001. p. 5-15.

21. Minayo, MCS. O desafio do Conhecimento: Pesquisa Qualitativa em Saúde. 2.ed. São Paulo: Hucitec/Abrasco; 1993.

22. Bardin L. Análise de Conteúdo. Lisboa: Edições 70; 1995.

23. Perrenoud P. Construir competências desde a escola. Porto Alegre: Artes Médicas Sul; 1999.

24. Vidal DE. A necessidade da pratica da criatividade e da melhoria dos relacionamentos interpessoais no processo ensino-aprendizagem: um estudo de caso [dissertação]. Florianópolis (SC): Engenharia da Produção, Universidade Federal de Santa Catarina; 2002. Mestrado em Engenharia de Produção.
25. Ramos MN. Qualificação, competências e certificação: visão educacional. In: Ministério da Saúde (BR). Humanizar cuidados de saúde: uma questão de competência. Brasília: Ministério da Saúde; 2001. p.17-26.

26. Lazarotto EM. Competências essenciais requeridas para o gerenciamento de Unidades Básicas de Saúde [dissertação]. Florianópolis (SC): Engenharia da Produção, Universidade Federal de Santa Catarina; 2001. Mestrado em Engenharia de Produção.

27. Maeda DY. Preceptoria no curso de residência em enfermagem: competências da enfermeira [dissertação]. Salvador (BA): Escola de Enfermagem, Universidade Federal da Bahia; 2006. Mestrado em Enfermagem.

28. Benjumea CC. Cuidado familiar en condiciones crónicas: una aproximación a la literatura. Texto Contexto Enfermagem 2004;13(1):137-46.

29. Marques CMS. Certificação de competências profissionais: o que o PROFAE está pensando e fazendo. In: Ministério da Saúde (BR). Humanizar cuidados de saúde: uma questão de competência. Brasília: Ministério da Saúde; 2001. p. 53-60.

30. Rehem CM. Certificação de competências em educação profissional: concepção e implementação. In: Ministério da Saúde (BR). Humanizar cuidados de saúde: uma questão de competência. Brasília: Ministério da Saúde; 2001. p. $39-43$

31. Heckert AL. Ética e Técnica: exercício e fabricações. In: Pinheiro R, Mattos RA, organizadores. Cuidar do cuidado: responsabilidade com a integralidade das ações de saúde. Rio de Janeiro: CEPESC:IMS/UERJ:ABRASCO; 2008. p.213-230.

32. Waldow VR. O cuidado na saúde: as relações entre o eu, o outro e o cosmos. Petrópolis- RJ: Vozes;2004.

33. Schraiber L. No encontro da técnica com a ética: o exercício de julgar e decidir no cotidiano do trabalho médico. Interf Comun Saúd Edu 1997; 1(1):123-40.

34. Prado ML, Reibnitz KS, Gelbcke FL. Aprendendo a cuidar: a sensibilidade como elemento plasmático para formação da profissional crítico-criativa em enfermagem. Texto Contexto Enferm 2006; 15(2):296-3. 